\title{
Hemeoxygenase-1 Mediates an Adaptive Response to Spermidine-Induced Cell Death in Human Endothelial Cells
}

\author{
Hana Yang, Seung Eun Lee, Gun-Dong Kim, Hye Rim Park, and Yong Seek Park \\ Department of Microbiology, School of Medicine, Kyung Hee University, No. 1 Hoegi-Dong, Dongdaemun-Gu, \\ Seoul 130-701, Republic of Korea \\ Correspondence should be addressed to Yong Seek Park; yongseek@khu.ac.kr
}

Received 5 April 2013; Revised 2 July 2013; Accepted 9 July 2013

Academic Editor: Hun-Taeg Chung

Copyright (c) 2013 Hana Yang et al. This is an open access article distributed under the Creative Commons Attribution License, which permits unrestricted use, distribution, and reproduction in any medium, provided the original work is properly cited.

Spermidine (SPD) is a ubiquitous polycation that is commonly distributed in living organisms. Intracellular levels of SPD are tightly regulated, and SPD controls cell proliferation and death. However, SPD undergoes oxidation in the presence of serum, producing aldehydes, hydrogen peroxide, and ammonia, which exert cytotoxic effect on cells. Hemeoxygenase-1 (HO-1) is thought to have a protective effect against oxidative stress. Upregulation of HO-1 in endothelial cells is considered to be beneficial in the cardiovascular disease. In the present study, we demonstrate that the ubiquitous polyamine, SPD, induces HO-1 in human umbilical vein endothelial cells (HUVECs). SPD-induced HO-1 expression was examined by Western blot and reverse transcriptionpolymerase chain reaction (RT-PCR). Involvement of reactive oxygen species, serum amine oxidase, PI3K/Akt signaling pathway, and transcription factor Nrf2 in the induction of HO-1 by SPD was also investigated. Furthermore, small interfering RNA knockdown of Nrf2 or HO-1 and treatment with the specific HO-1 inhibitor ZnPP exhibited a noteworthy increase of death of SPDstimulated HUVECs. In conclusion, these results suggest that SPD induces PI3K/Akt-Nrf2-mediated HO-1 expression in human endothelial cells, which may have a role in cytoprotection of the cells against oxidative stress-induced death.

\section{Introduction}

Polyamines are common cell components among almost all types of organisms, which regulate cell proliferation and differentiation. The function of polyamines has been studied at the molecular level. Polyamines modulate the function of RNA, DNA, and protein by promoting their stability and synthesis [1]. Polyamine content within cells is tightly regulated and retained both by polyamine biosynthesis and by polyamine transport according to needs [2]. For example, it is reported that proliferative stimuli, such as DNA synthesis, trigger uptake and/or biosynthesis of polyamines [3]. There are three polyamines in eukaryotic cells: putrescine, spermidine, and spermine. As long as the polyamine pool is strictly regulated also by interconversion, excessive polyamine accumulation following exogenous addition may exert a different effect on cells. Extracellular polyamines first undergo oxidation by serum amine oxidase, producing aminoaldehydes, such as aminomonoaldehyde [ $N^{\prime}$-(4-aminobutyl)-aminopropion- aldehyde] and a dialdehyde [ $N N^{\prime}$-bis-(3-propionaldehyde)1,4-diaminobutane] [4]. These aminoaldehydes and other oxidation products (e.g., $\mathrm{H}_{2} \mathrm{O}_{2}$ and $\mathrm{NH}_{3}$ ) show cytotoxicity in several cell types $[5,6]$. Due to the oxidation by serum amine oxidase, the endothelium would be the first tissue with which the products of polyamine oxidation interact.

Vascular injury generally refers to structural and functional impairment of endothelium including damage at the cellular level. It plays a key role in the pathogenesis of various cardiovascular diseases, such as atherosclerosis, diabetic complications, and hypertension. Hemeoxygenase $(\mathrm{HO})$ is one of the cytoprotective proteins that could confer a beneficial effect in vasculature. HO originally functions as a rate-limiting enzyme in heme degradation, yielding carbon monoxide (CO), iron, and biliverdin as the end products. $\mathrm{HO}-1, \mathrm{HO}-2$, and $\mathrm{HO}-3$ are isoforms of $\mathrm{HO}$ in mammals $[7,8]$. In particular, the role of hemeoxygenase1 (HO-1) as a protective enzyme is well known. Its antioxidant, antiapoptotic, and anti-inflammatory effects have been extensively studied $[9,10]$. HO-1 is a stress-inducible 


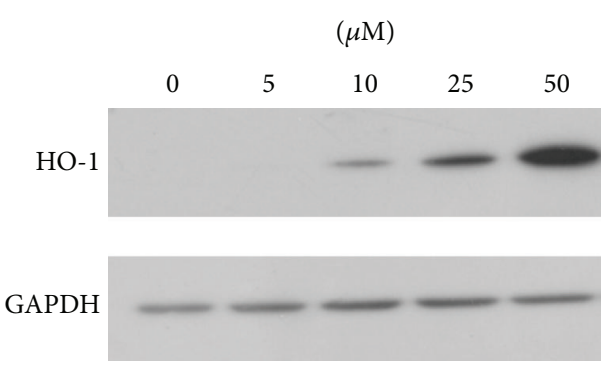

(a)

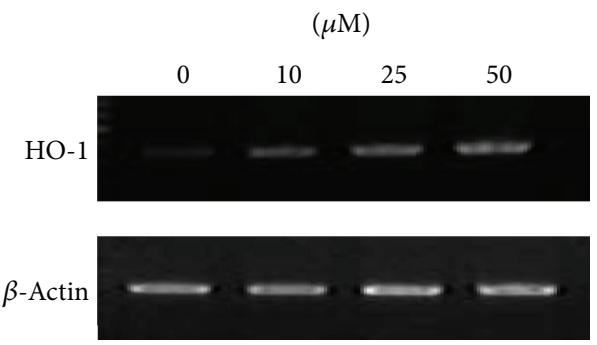

(c) (h)

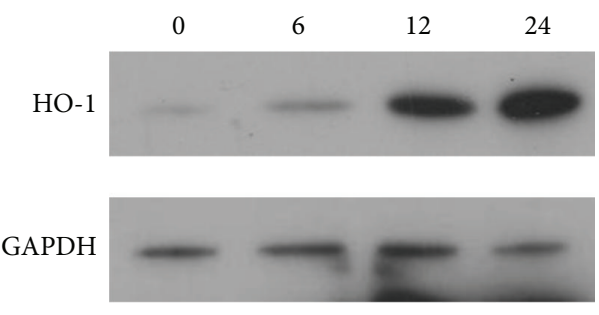

(b)

(h)

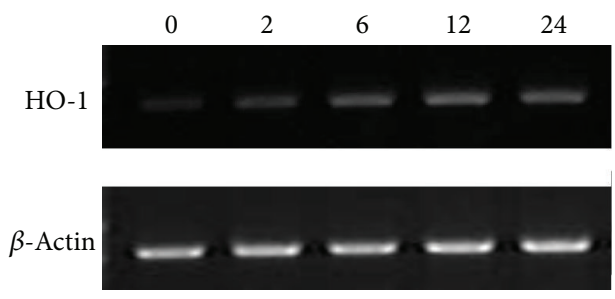

(d)

FIgUre 1: Induction of HO-1 by SPD in HUVECs. After treatment of HUVECs with various concentrations of SPD (a) at various time intervals (b), cell lysates were prepared, and $20 \mu \mathrm{g}$ samples of proteins were subjected to Western blotting using the anti-HO-1 antibody, and anti-glyceraldehyde 3-phosphate dehydrogenase (GAPDH) antibody as a loading control. Representative data from three independent experiments are shown. After treatment of HUVECs with various concentrations of SPD (c) at various time intervals (d), cell lysates were prepared. The total RNA was extracted and analyzed by RT-PCR. The amplified RT-PCR product was visualized on $1 \%$ agarose gel. Representative data from three independent experiments are shown.

protein. Various stimuli, such as thiol scavengers, ultraviolet radiation, and oxidative stress, lead to $\mathrm{HO}-1$ upregulation. Usually, these types of stimuli generate reactive oxygen species (ROS), which may cause an adaptive response to HO1. HO-1 is one of the phase II detoxifying and antioxidant enzymes that are modulated by the nuclear factor-erythroid 2-related factor 2 (Nrf2)/Kelch-like ECH associating protein 1 (Keap1)/antioxidant responsive element (ARE) transcription factor system [11]. Inhibition of Keap1 activity allows Nrf2 translocation from cytoplasm to nucleus, and then Nrf2 binds to the ARE region to activate expression of phase II gene, such as HO-1 [12]. Upregulation of HO-1 has protective effects in various cell types due to the activities of its final products, such as $\mathrm{CO}$ and/or bilirubin $[13,14]$. HO-1 is also known to exert a beneficial effect in several clinically relevant diseases, especially vascular disease, including atherosclerosis, diabetes, and hypertension [15]. HO-1, which is highly expressed in vascular tissues under certain conditions, protects against cardiovascular diseases and contributes to sustain the health of the vascular system. In human endothelial cells, lack of HO-1 leads to endothelial damage induced by tumor necrosis factor- $\alpha$ and interleukin- $\alpha$ [16]. These results suggest that HO-1 may play an important role in the human cardiovascular system.

In this study, we demonstrate that polyamine SPD induces HO-1 expression in human endothelial cells and the association of serum amine oxidase, ROS, and phosphatidylinositol 3-kinase (PI3K)/Akt-Nrf2-ARE signaling pathway in the upregulation of HO-1 by SPD.

\section{Materials and Methods}

2.1. Materials. M199 medium and fetal bovine serum (FBS) were purchased from WELGENE (Daegu, Korea). TRIzol reagent was supplied by Invitrogen (Carlsbad, CA, USA). Spermidine (SPD), aminoguanidine (AG), Nacetyl cysteine (NAC), MTT [3-(4,5-dimethylthiazol-2-yl)2,5-diphenyltetrazoliumbromide], and dimethylsulphoxide (DMSO) were provided by Sigma Chemical (St. Louis, MO, USA). ExGen 500 reagent was obtained from Fermentas (Hanover, MD, USA). Anti-Nrf2 and anti-Lamin B antibodies were purchased from Santa Cruz Biotechnology (Santa Cruz, CA, USA). Anti-HO-1 antibody was obtained from Epitomics (Burlingame, CA, USA). Anti-GAPDH antibody was supplied by AbFrontier (Seoul, Korea). All other chemicals and reagents were of analytical grade.

2.2. Cell Culture. HUVECs were maintained in M199 medium and supplemented with $10 \%$ fetal bovine serum, $1 \%$ penicillin and streptomycin, $10 \mathrm{ng} / \mathrm{mL}$ human fibroblast growth factor, and $18 \mathrm{mU} / \mathrm{mL}$ heparin. The cells were incubated at $37^{\circ} \mathrm{C}$ under $5 \% \mathrm{CO}_{2}$ atmosphere. HUVECs were grown to approximately $80 \%$ confluence, maintained with the fresh medium previously described, and subcultured every 2 3 days [17]. The cells were used within passages 4-9 during these experiments.

2.3. Western Blot Analysis. We applied $20 \mu \mathrm{g}$ of the whole cell lysate proteins to each lane and analyzed them with Western 
blot. Western blot analysis was performed using monoclonal antibody against hemeoxygenase-1 (HO-1), anti-Nrf2, antiLamin B and anti-glyceraldehyde-3-phosphate dehydrogenase $(\mathrm{GAPDH})$. Horseradish peroxidase-conjugated anti-IgG antibodies were used as the secondary antibodies to detect the previously mentioned protein bands by enhanced chemiluminescence WESTSAVE-Up (AbFrontier, Seoul, Korea) [18].

2.4. RNA Isolation and Reverse Transcriptase-Polymerase Chain Reaction (RT-PCR). Reverse transcription was performed as previously described [19]. The primer sequences for human HO-1 were $5^{\prime}$-ACATCTATGTGGCCCTGGAG$3^{\prime}$ (forward) and $5^{\prime}$-TGTTGGGGAAGGTGAAGAAG-3' (reverse). The amplified products were resolved by $1 \%$ agarose gel electrophoresis, stained with ethidium bromide, and photographed under ultraviolet light.

2.5. Measurement of ARE Promoter Activity. EpRE/AREluciferase (EpRE/ARE-Luc) reporter plasmid was a generous gift from Dr. R. K. Park, (Wonkwang University, Korea). EpRE/ARE-Luc was generated by transfer of the enhancer 2 (E2) and minimal promoter (MP) sequences into the luciferase reporter plasmid pGL3-Basic. ARE promoter activity was determined as previously described [20].

2.6. Terminal Deoxynucleotidyl Transferase-Mediated dUTP Nick-End Labeling (TUNEL) Assay. To measure DNA fragmentation, the commercially available in situ death detection kit (Roche Diagnostics, Mannheim, Germany) was used. The cells were examined by fluorescence microscopy and photographed as previously described [21]. TUNEL-positive cells were determined by counting at least 100 cells in 3 randomly chosen fields and by expressing them as a percentage of the total number of cells counted.

2.7. Statistical Analysis. Statistical significance was estimated by Student's $t$-test, and the results were expressed as mean \pm SD.

\section{Results}

3.1. HO-1 Induction by SPD in HUVECs. Upregulation of HO-1 expression is thought to be a cellular stress response and to play an important role in protecting cells. Presently, we investigated the effect of various concentrations of SPD on HO-1 induction. Cells were incubated with the indicated concentrations of SPD for $24 \mathrm{~h}$. SPD increased HO1 protein expression in a concentration-dependent manner (Figure 1(a)). Treatment of cells with $50 \mu \mathrm{M}$ SPD also resulted in a time-dependent increase in $\mathrm{HO}-1$ protein expression in HUVECs (Figure 1(b)). SPD increased the HO-1 mRNA level as well. After incubation with various concentrations of SPD for $8 \mathrm{~h}$, the HO-1 mRNA level increased in a concentrationdependent manner (Figure 1(c)). SPD-treated HUVECs also showed increased HO-1 mRNA levels in a time-dependent manner (Figure 1(d)).
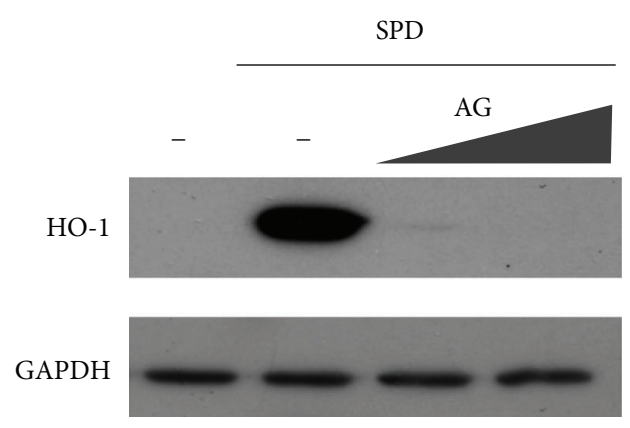

(a)
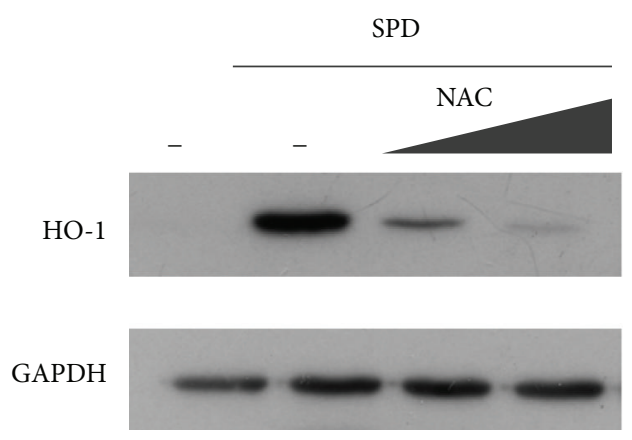

(b)

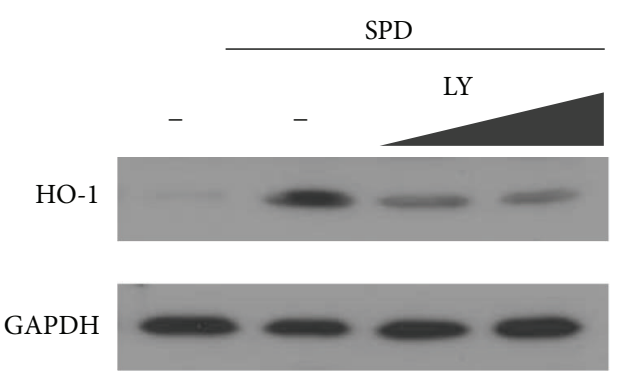

(c)

FIGURE 2: Blockage of SPD-induced HO-1 protein expression by aminoguanidine, NAC, or PI3K/Akt inhibitor. Cells were pretreated with aminoguanidine (a), NAC (b), or LY 294002 (PI3K/Akt inhibitor) (c) $1 \mathrm{~h}$ prior to the treatment of SPD. After $24 \mathrm{~h}$ incubation, cell lysates were prepared, and $20 \mu \mathrm{g}$ samples of proteins were subjected to Western blotting, using anti-HO-1 antibody and antiGAPDH.

3.2. Blockage of SPD-Induced HO-1 Protein Expression by Aminoguanidine, NAC, or PI3K/Akt Inhibitor. Polyamines, including SPD, are deaminated by bovine serum amine oxidase under in vitro conditions [22], generating aldehydes, $\mathrm{H}_{2} \mathrm{O}_{2}$, and ammonia. The polyamine oxidation is inhibited by the serum amine oxidase inhibitor, aminoguanidine (AG) [23]. Therefore, we tested whether the deamination of SPD by oxidase in serum could affect HO-1 induction in human endothelial cells. Cells were pretreated with 0.1 or $1 \mathrm{mM}$ $\mathrm{AG}$ and then treated with $50 \mu \mathrm{M}$ SPD. Induction of $\mathrm{HO}-1$ by SPD was completely eliminated by the pretreatment of AG (Figure 2(a)). These results indicate the involvement of polyamine oxidation in the induction of HO-1 by SPD.

The polyamine oxidation products are known to generate reactive oxygen species (ROS), the signaling molecules in 
$(\mu \mathrm{M})$

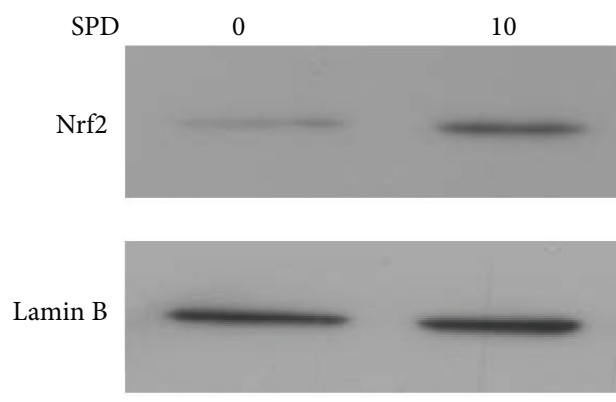

(a)

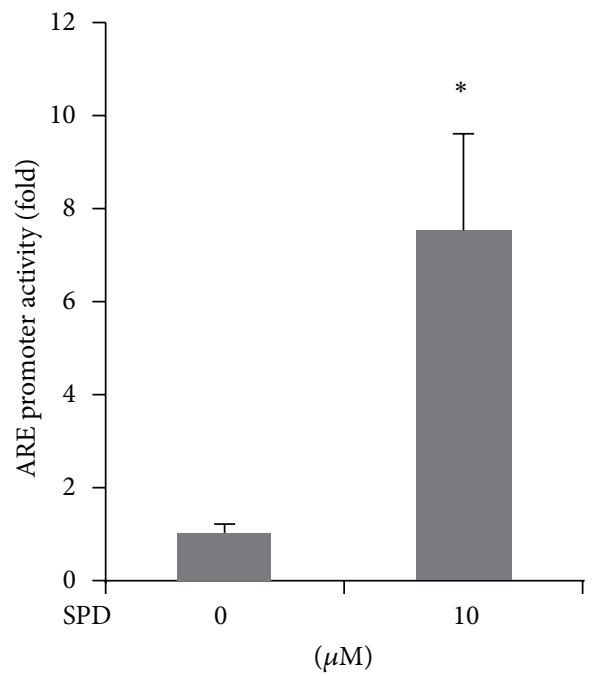

(b)

FIGURE 3: Nrf2 nuclear translocation and ARE-luciferase reporter activity induced by SPD. Cells were treated with SPD at the indicated concentration for $4 \mathrm{~h}$. (a) Nuclear extracts were prepared, and $10 \mu \mathrm{g}$ samples of proteins were subjected to Western blotting, using an anti-Nrf2 antibody and an anti-Lamin B (a marker of nuclear protein) antibody. (b) Cells were transfected with an ARE-luciferase construct. After transfection, the cells were treated with $10 \mu \mathrm{M}$ SPD for $6 \mathrm{~h}$, and the lysates were mixed with a luciferase substrate. A luminometer was used for measurement of luciferase activity. Data are the mean $\pm \mathrm{SD}$ of quintuplicate experiments. ${ }^{*} P<0.05$ versus control.

mediating responses to various stimuli [24]. Since ROS have a role in the induction of $\mathrm{HO}-1$ by several compounds $[25,26]$, we examined whether the HO-1 induction by SPD was dependent on ROS by using N-acetyl cysteine (NAC), a ROS scavenger. Pretreatment with NAC abolished HO-1 expression in SPD-stimulated HUVECs in a dose-dependent manner (Figure 2(b)). This implies the involvement of ROS in the induction of HO-1 by SPD in HUVECs.

To determine the upstream signaling pathway involved in SPD-mediated HO-1 induction, the effects of the specific inhibitors of the PI3K/Akt pathways on HO-1 expression were evaluated. Inhibitors of the PI3K/Akt pathways reduced SPD-induced HO-1 expression (Figure 2(c)). The observation
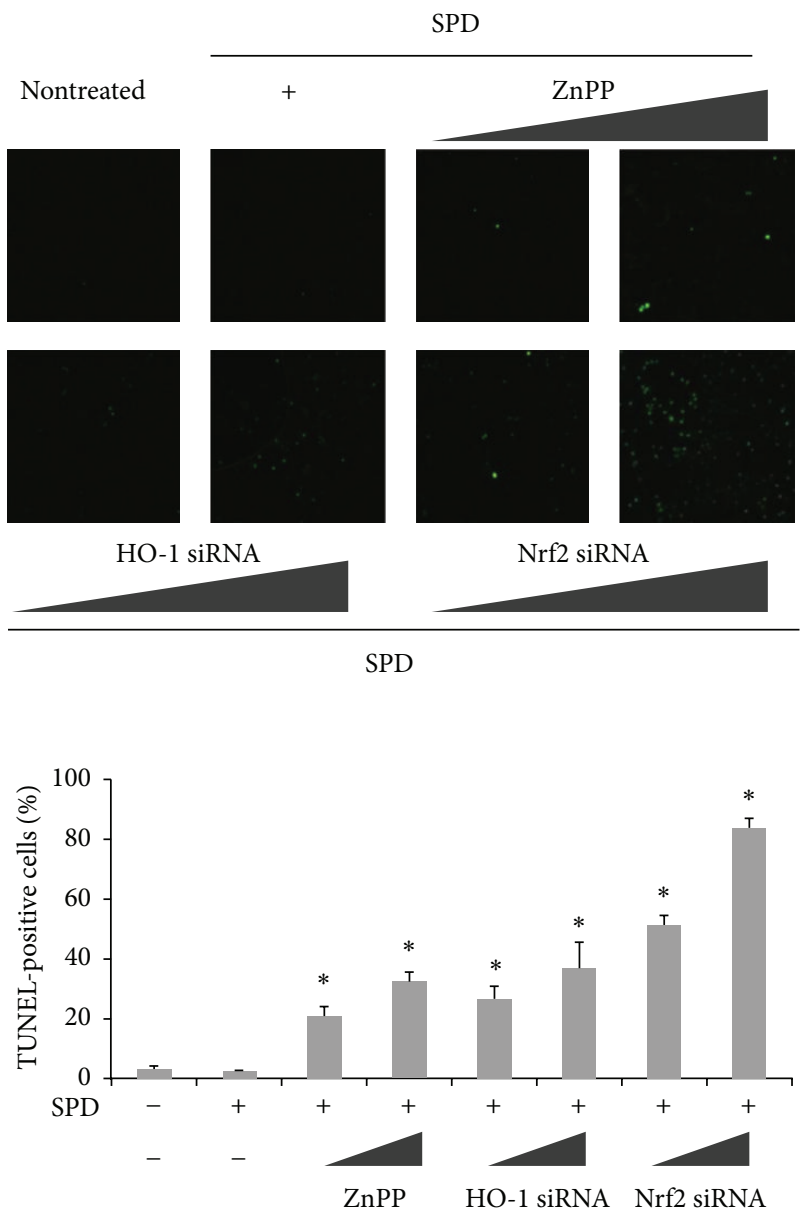

FIGURE 4: Effect of SPD-induced HO-1 inhibition on cell death. Cells were incubated in the absence or presence of $\mathrm{ZnPP}$, siRNA of HO1 or Nrf2 for $24 \mathrm{~h}$ before the indicated tests were performed. SPDstimulated HUVECs were pretreated with or without ZnPP, HO-1 or Nrf2 siRNA. Protective effect of HO-1 induction on cell death was determined by in situ terminal nick-end labeling (TUNEL). Representative images illustrate fluorescent TUNEL (green) staining of cells cultured for $24 \mathrm{~h}$ before the indicated tests were performed. The percentage of TUNEL-positive cells per total cell count in each sample was calculated. At least 100 cells from three random fields were counted in each experiment. Data are the mean \pm SD of triplicate experiments. ${ }^{*} P<0.05$ versus control.

supported a role for PI3K/Akt signaling in SPD-mediated HO-1 induction in HUVECs.

3.3. Nrf2 Nuclear Translocation and ARE-Luciferase Reporter Activity Induced by SPD. HO-1 belongs to a family of the genes encoding phase II detoxifying and antioxidant enzymes and is widely distributed in mammalian tissues. It is modulated by the Nrf2/Keap1 transcription factor system including Nrf2 binding to ARE in the nucleus [11]. Appropriately, we attempted to determine whether SPD could activate Nrf2 in association with HO-1 upregulation. For this, the cells were treated with $10 \mu \mathrm{M}$ of SPD for $4 \mathrm{~h}$, and the nuclear fractions were extracted for the preparation of nuclear proteins. SPD treatment in HUVECs stimulated Nrf2 accumulation in the 


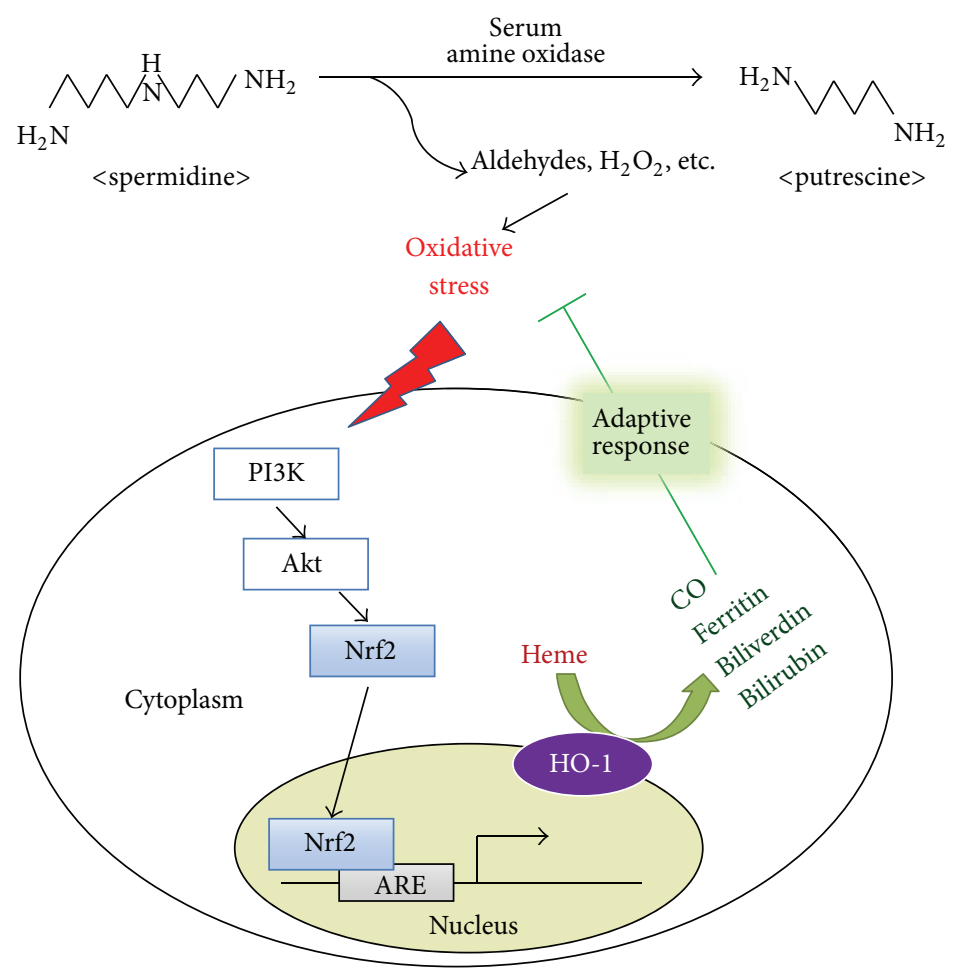

FIGURE 5: A scheme showing a proposed cytoprotective mechanism against SPD-induced oxidative stress by HO-1 induction. During the degradation of spermidine (SPD) to putrescine by serum amine oxidase, aldehydes, $\mathrm{H}_{2} \mathrm{O}_{2}$, and $\mathrm{NH}_{3}$ are produced, which induce oxidative stress. It triggers Nrf2 translocation and ARE binding through PI3K/Akt signaling. Nrf2 activation upregulates HO-1 expression. HO-1 catalyzed heme to biliverdin and bilirubin, with the concurrent release of bioactive molecules, such as ferritin and CO. Heme metabolites exert adaptive response and protect cells against SPD-induced oxidative stress.

nucleus (Figure 3(a)). In addition to this, we measured ARE promoter activity using luciferase reporter system. Cells were transiently transfected with ARE luciferase reporter plasmids and treated with $10 \mu \mathrm{M}$ SPD for $6 \mathrm{~h}$, and luciferase activity was determined. As expected, treatment with SPD at the smallest concentrations $(10 \mu \mathrm{M})$ increased ARE promoter activity by approximately 8 folds (Figure $3(\mathrm{~b}) ;{ }^{*} P<0.05$ ). These results indicate the association with $\mathrm{Nrf} 2$ activation in regulation of SPD-induced HO-1 expression in HUVECs.

\subsection{Effect of SPD-Induced HO-1 Inhibition on Cell Death.} HO-1 is a well-known cytoprotective enzyme inhibiting cell death in different cell types $[20,27,28]$. To further determine whether the increased level of HO-1 enhanced by SPD confers cytoprotection, SPD-stimulated cells were pre-incubated with or without a specific HO-1, inhibitor, ZnPP, HO-1, or Nrf2 siRNA, and the presence of dead cells was assessed by in situ terminal nick-end labeling (TUNEL) staining, which is widely used in detecting DNA fragmentation in situ. SPDtreated cells showed little difference in nontreated cells, while pharmacological inhibition of HO-1, or the transcription factor Nrf2 resulted in a notable increase in the proportion of TUNEL-positive cells (Figure 4). These results suggest that HO-1 may exert a protective effect against SPD-stimulated cell death.

\section{Discussion}

The present study demonstrates that the polyamine SPD clearly induces HO-1 in human endothelial cells through activation of Nrf2. Presence of ROS scavenger or serum amine oxidase inhibitor abrogates the increase of HO-1.

Although polyamines are essential for cell metabolism, the excessive accumulation within cells by exogenous addition of polyamine or high extracellular concentrations can result in toxic effects. Interconversion of polyamine is one of the ways to modulate polyamine pool homeostasis. Polyamines are synthesized from L-arginine via L-ornithine, or L-methionine by specific enzyme reactions. Putrescine is formed from L-ornithine by ornithine decarboxylase, and this combines with decarboxylated $S$-adenosylmethionine originating from L-methionine to produce spermidine in a reaction catalyzed by spermidine synthase [29]. Spermine is formed from spermidine by spermine synthase. Spermidine and spermine also undergo the retroconversion process by the action of spermidine/spermine N1-acetyltransferase and polyamine oxidase [30] and possibly spermine oxidase, in the direct conversion of spermine back to spermidine without acetylation [31]. During the catabolic process, toxic metabolites of polyamine are generated, including $\mathrm{H}_{2} \mathrm{O}_{2}$, ammonia, and aminoaldehyde(s). Moreover, after spermidine is oxidized by amine oxidase, an $\alpha, \beta$-unsaturated aldehyde, acrolein, is formed by further spontaneous degradation 
[32]. Acrolein, which is highly reactive, can induce $\mathrm{HO}-1$ expression as well as $\mathrm{H}_{2} \mathrm{O}_{2}$ [33] and ammonia [34]. Especially, the induction of HO-1 by toxic substances is thought to be an adaptive response [20].

HO-1 contributes to the cellular defense against oxidative stress generated by ROS. Increase of HO-1 activity protects cells from oxidative damage-induced cell death $[21,35]$. In addition, since HO-1 is highly expressed in vascular tissues, it protects against vascular injury and confers cytoprotection in the circulation. The beneficial effect of HO-1 is mediated by the actions of its metabolic byproducts, carbon monoxide, and bilirubin. Carbon monoxide modulates blood fluidity and flow by regulating vasomotor tone, vascular smooth muscle cell proliferation, and platelet agglutination [36]. Bilirubin preserves endothelial cell integrity, prevents cell death, and increases vascular reactivity [37].

Transcriptional activation of the HO-1 gene is mediated by Nrf2 [38], which is initiated by translocation of Nrf2 into the nucles after electrophilic modification of Keap1 for dissociation of the Nrf2/Keap1 complex [11]. Nrf2 in nucleus binds to ARE in the promoter region of its target genes, the phase II detoxification and antioxidant genes, including $\mathrm{NAD}(\mathrm{P}) \mathrm{H}$ :quinone oxidoreductase 1, glutathione S-transferases, peroxiredoxin-1, and $\gamma$-glutamylcysteine ligase. Several oxidants induce the phase II detoxification and antioxidant gene expression via Nrf2 activation [39]. In particular, Nrf2 activation bestows cytoprotection to vascular tissues by induction of antioxidant gene expression and/or suppression of redox-sensitive inflammatory genes [40, 41]. The Nrf2/ARE pathway is involved in SPD-induced HO-1 expression, as some polyamines can indeed activate other Nrf2-related genes [42].

In this study, we demonstrated that the polyamine SPD, possibly via its oxidized metabolites, induces HO-1 in human primary endothelial cells in association with Nrf2/ARE signaling system, and induction of HO-1 contributes to cell survival in oxidative stress-induced cell death (see Figure 5). This study provides evidence that the induction of HO-1 may have a role of cellular defense against the cytotoxic effects of SPD oxidation in vascular endothelial cells.

\section{Conflict of Interests}

The authors have no conflict of interests to declare.

\section{Acknowledgment}

This study was supported by a grant from the Korean Health Technology R\&D Project, Ministry of Health \& Welfare, Republic of Korea (A111834).

\section{References}

[1] K. Igarashi and K. Kashiwagi, "Polyamines: mysterious modulators of cellular functions," Biochemical and Biophysical Research Communications, vol. 271, no. 3, pp. 559-564, 2000.

[2] N. Seiler, J. G. Delcros, and J. P. Moulinoux, "Polyamine transport in mammalian cells. An update," The International
Journal of Biochemistry and Cell Biology, vol. 28, no. 8, pp. 843861, 1996.

[3] Y. Kakinuma, K. Hoshino, and K. Igarashi, "Characterization of the inducible polyamine transporter in bovine lymphocytes," European Journal of Biochemistry, vol. 176, no. 2, pp. 409-414, 1988.

[4] C. W. Tabor, H. Tabor, and U. Bachrach, "Identification of the aminoaldehydes produced by the oxidation of spermine and spermidine with purified plasma amine oxidase," The Journal of Biological Chemistry, vol. 239, no. 7, pp. 2194-2203, 1964.

[5] E. Schenkel, J. G. Dubois, M. Helson-Cambier, and M. Hanocq, "Cytotoxicity of polyamines to Amoeba proteus: role of polyamine oxidase," Cell Biology and Toxicology, vol. 12, no. 1, pp. 1-9, 1996.

[6] A. Pledgie, Y. Huang, A. Hacker et al., "Spermine oxidase $\mathrm{SMO}\left(\mathrm{PAOh}_{1}\right)$, not $N^{1}$-acetylpolyamine oxidase $\mathrm{PAO}$, is the primary source of Cytotoxic $\mathrm{H}_{2} \mathrm{O}_{2}$ in polyamine analoguetreated human breast cancer cell lines," Journal of Biological Chemistry, vol. 280, no. 48, pp. 39843-39851, 2005.

[7] M. D. Maines, G. M. Trakshel, and R. K. Kutty, "Characterization of two constitutive forms of rat liver microsomal heme oxygenase. Only one molecular species of the enzyme is inducible," The Journal of Biological Chemistry, vol. 261, no. 1, pp. 411-419, 1986.

[8] W. K. McCoubrey Jr., T. J. Huang, and M. D. Maines, "Isolation and characterization of a cDNA from the rat brain that encodes hemoprotein heme oxygenase-3," European Journal of Biochemistry, vol. 247, no. 2, pp. 725-732, 1997.

[9] Y.-J. Surh, J. K. Kundu, M.-H. Li, H.-K. Na, and Y.-N. Cha, "Role of Nrf2-mediated heme oxygenase-1 upregulation in adaptive survival response to nitrosative stress," Archives of Pharmacal Research, vol. 32, no. 8, pp. 1163-1176, 2009.

[10] M. Zheng, S. K. Kim, Y. Joe et al., "Sensing endoplasmic reticulum stress by protein kinase RNA-like endoplasmic reticulum kinase promotes adaptive mitochondrial DNA biogenesis and cell survival via heme oxygenase-1/carbon monoxide activity," FASEB Journal, vol. 26, no. 6, pp. 2558-2568, 2012.

[11] M. Kobayashi and M. Yamamoto, "Molecular mechanisms activating the Nrf2-Keap1 pathway of antioxidant gene regulation," Antioxidants and Redox Signaling, vol. 7, no. 3-4, pp. 385-394, 2005.

[12] K. Itoh, N. Wakabayashi, Y. Katoh et al., "Keap1 represses nuclear activation of antioxidant responsive elements by Nrf2 through binding to the amino-terminal Neh2 domain," Genes and Development, vol. 13, no. 1, pp. 76-86, 1999.

[13] D. E. Barañano, M. Rao, C. D. Ferris, and S. H. Snyder, "Biliverdin reductase: a major physiologic cytoprotectant," Proceedings of the National Academy of Sciences of the United States of America, vol. 99, no. 25, pp. 16093-16098, 2002.

[14] M. Constantin, A. J. Choi, S. M. Cloonan, and S. W. Ryter, "Therapeutic potential of heme oxygenase-1/carbon monoxide in lung disease," International Journal of Hypertension, vol. 2012, Article ID 859235, 19 pages, 2012.

[15] J. Deshane, M. Wright, and A. Agarwal, "Heme oxygenase-1 expression in disease states," Acta Biochimica Polonica, vol. 52, no. 2, pp. 273-284, 2005.

[16] C. M. Terry, J. A. Clikeman, J. R. Hoidal, and K. S. Callahan, "Effect of tumor necrosis factor- $\alpha$ and interleukin- $1 \alpha$ on heme oxygenase-1 expression in human endothelial cells," American Journal of Physiology, vol. 274, no. 3, pp. H883-H891, 1998.

[17] S. I. Jeong, S. E. Lee, H. Yang, C.-S. Park, Y.-H. Jin, and Y. S. Park, "Effect of $\alpha, \beta$-unsaturated aldehydes on endothelial cell growth 
in bacterial cellulose for vascular tissue engineering," Molecular \& Cellular Toxicology, vol. 8, no. 2, pp. 119-126, 2012.

[18] S. E. Lee, H. Yang, S. I. Jeong, Y. H. Jin, C. S. Park, and Y. S. Park, "Induction of heme oxygenase-1 inhibits cell death in crotonaldehyde-stimulated HepG2 cells via the PKC- $\delta$-p38 Nrf2 pathway," PLoS ONE, vol. 7, no. 7, article e41676, 2012.

[19] Y. S. Park, J. Kim, Y. Misonou et al., "Acrolein induces cyclooxygenase-2 and prostaglandin production in human umbilical vein endothelial cells: roles of p38 MAP kinase," Arteriosclerosis, Thrombosis, and Vascular Biology, vol. 27, no. 6, pp. 1319-1325, 2007.

[20] S. E. Lee, S. I. Jeong, G.-D. Kim et al., "Upregulation of heme oxygenase- 1 as an adaptive mechanism for protection against crotonaldehyde in human umbilical vein endothelial cells," Toxicology Letters, vol. 201, no. 3, pp. 240-248, 2011.

[21] H. Yang, S. E. Lee, S. I. Jeong, C.-S. Park, Y.-H. Jin, and Y. S. Park, "Up-regulation of heme oxygenase-1 by Korean red ginseng water extract as a cytoprotective efect in human endothelial cells," Journal of Ginseng Research, vol. 35, no. 3, pp. 352-359, 2011.

[22] W. A. Gahl and H. C. Pitot, "Polyamine degradation in foetal and adult bovine serum," Biochemical Journal, vol. 202, no. 3, pp. 603-611, 1982.

[23] H. Tamura, K. Horiike, H. Fukuda, and T. Watanabe, "Kinetic studies on the inhibition mechanism of diamine oxidase from porcine kidney by aminoguanidine," The Journal of Biochemistry, vol. 105, no. 2, pp. 299-306, 1989.

[24] E. Agostinelli, G. Arancia, L. D. Vedova et al., "The biological functions of polyamine oxidation products by amine oxidases: perspectives of clinical applications," Amino Acids, vol. 27, no. 3-4, pp. 347-358, 2004.

[25] W. Y. Seo, A. R. Goh, S. M. Ju et al., "Celastrol induces expression of heme oxygenase-1 through ROS/Nrf2/ARE signaling in the HaCaT cells," Biochemical and Biophysical Research Communications, vol. 407, no. 3, pp. 535-540, 2011.

[26] J.-Y. Kim, H.-J. Cho, J.-J. Sir et al., "Sulfasalazine induces haem oxygenase-1 via ROS-dependent Nrf2 signalling, leading to control of neointimal hyperplasia," Cardiovascular Research, vol. 82, no. 3, pp. 550-560, 2009.

[27] S. E. Lee, S. I. Jeong, H. Yang et al., "Extract of Salvia miltiorrhiza (Danshen) induces Nrf2-mediated heme oxygenase-1 expression as a cytoprotective action in RAW 264.7 macrophages," Journal of Ethnopharmacology, vol. 139, no. 2, pp. 541-548, 2012.

[28] Y. Joe, M. Zheng, H. J. Kim et al., "Salvianolic acid B exerts vasoprotective effects through the modulation of heme oxygenase-1 and arginase activities," Journal of Pharmacology and Experimental Therapeutics, vol. 341, no. 3, pp. 850-858, 2012.

[29] H. M. Wallace, A. V. Fraser, and A. Hughes, "A perspective of polyamine metabolism," Biochemical Journal, vol. 376, part 1, pp. 1-14, 2003.

[30] F. N. Bolkenius and N. Seiler, "Acetylderivatives as intermediates in polyamine catabolism," International Journal of Biochemistry, vol. 13, no. 3, pp. 287-292, 1981.

[31] S. Vujcic, P. Diegelman, C. J. Bacchi, D. L. Kramer, and C. W. Porter, "Identification and characterization of a novel flavin-containing spermine oxidase of mammalian cell origin," Biochemical Journal, vol. 367, part 3, pp. 665-675, 2002.

[32] R. A. Alarcon, "Acrolein. IV. Evidence for the formation of the cytotoxic aldehyde acrolein from enzymatically oxidized spermine or spermidine," Archives of Biochemistry and Biophysics, vol. 137, no. 2, pp. 365-372, 1970.
[33] K.-S. Min, H.-J. Lee, S.-H. Kim et al., "Hydrogen peroxide induces heme oxygenase-1 and dentin sialophosphoprotein mRNA in human pulp cells," Journal of Endodontics, vol. 34, no. 8, pp. 983-989, 2008.

[34] U. Warskulat, B. Görg, H.-J. Bidmon, H. W. Müller, F. Schliess, and D. Häussinger, "Ammonia-induced heme oxygenase-1 expression in cultured rat astrocytes and rat brain in vivo," Glia, vol. 40, no. 3, pp. 324-336, 2002.

[35] J. M. Woo, D. Y. Shin, S. J. Lee et al., "Curcumin protects retinal pigment epithelial cells against oxidative stress via induction of heme oxygenase-1 expression and reduction of reactive oxygen," Molecular Vision, vol. 18, no. 94-95, pp. 901-908, 2012.

[36] W. Durante and A. I. Schafer, "Carbon monoxide and vascular cell function (review)," International Journal of Molecular Medicine, vol. 2, no. 3, pp. 255-262, 1998.

[37] Y.-M. Kim, H.-O. Pae, J. E. Park et al., "Heme oxygenase in the regulation of vascular biology: from molecular mechanisms to therapeutic opportunities," Antioxidants and Redox Signaling, vol. 14, no. 1, pp. 137-167, 2010.

[38] J. Alam, D. Stewart, C. Touchard, S. Boinapally, A. M. K. Choi, and J. L. Cook, "Nrf2, a cap'n'collar transcription factor, regulates induction of the heme oxygenase-1 gene," Journal of Biological Chemistry, vol. 274, no. 37, pp. 26071-26078, 1999.

[39] S. E. Purdom-Dickinson, E. V. Sheveleva, H. Sun, and Q. M. Chen, "Translational control of Nrf2 protein in activation of antioxidant response by oxidants," Molecular Pharmacology, vol. 72, no. 4, pp. 1074-1081, 2007.

[40] G. E. Mann, J. Niehueser-Saran, A. Watson et al., "Nrf2/ARE regulated antioxidant gene expression in endothelial and smooth muscle cells in oxidative stress: implications for atherosclerosis and preeclampsia," Sheng Li Xue Bao, vol. 59, no. 2, pp. 117-127, 2007.

[41] X.-L. Chen, G. Dodd, S. Thomas et al., "Activation of Nrf2/ARE pathway protects endothelial cells from oxidant injury and inhibits inflammatory gene expression," American Journal of Physiology, vol. 290, no. 5, pp. H1862-H1870, 2006.

[42] M.-K. Kwak, T. W. Kensler, and R. A. Casero Jr., "Induction of phase 2 enzymes by serum oxidized polyamines through activation of Nrf2: effect of the polyamine metabolite acrolein," Biochemical and Biophysical Research Communications, vol. 305, no. 3, pp. 662-670, 2003. 


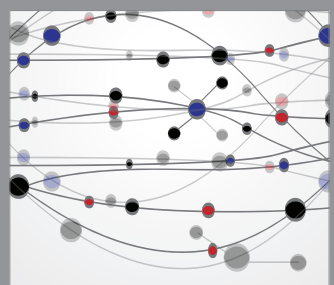

The Scientific World Journal
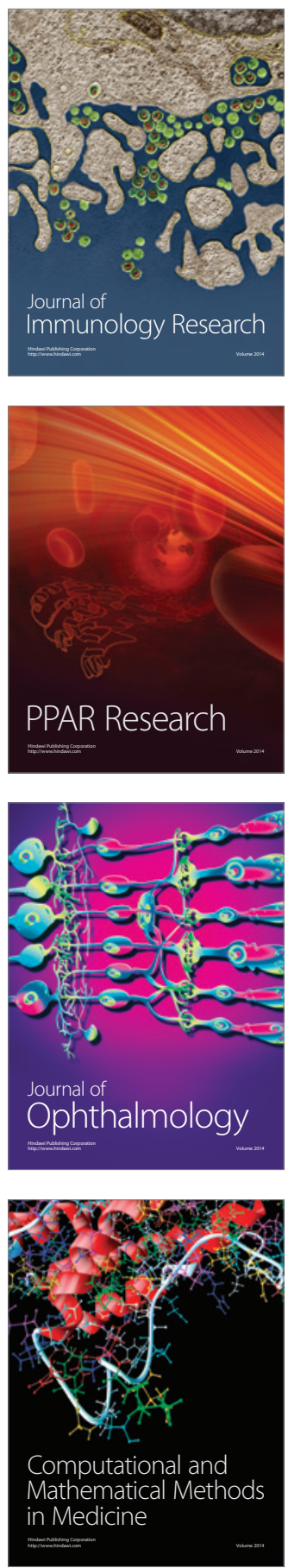

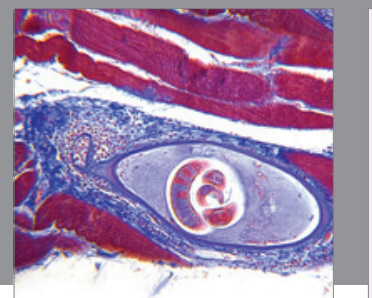

Gastroenterology

Research and Practice
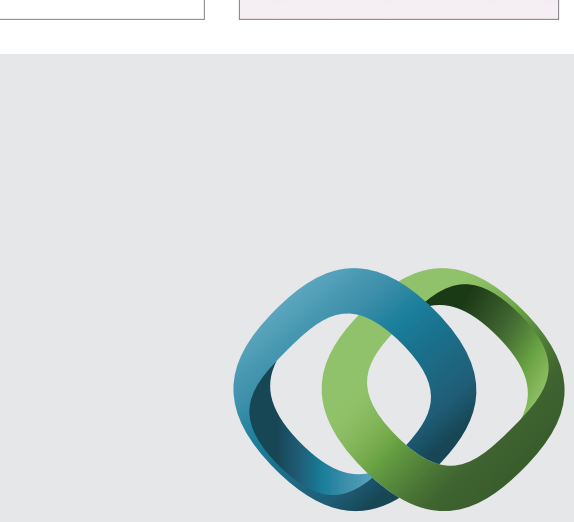

\section{Hindawi}

Submit your manuscripts at

http://www.hindawi.com
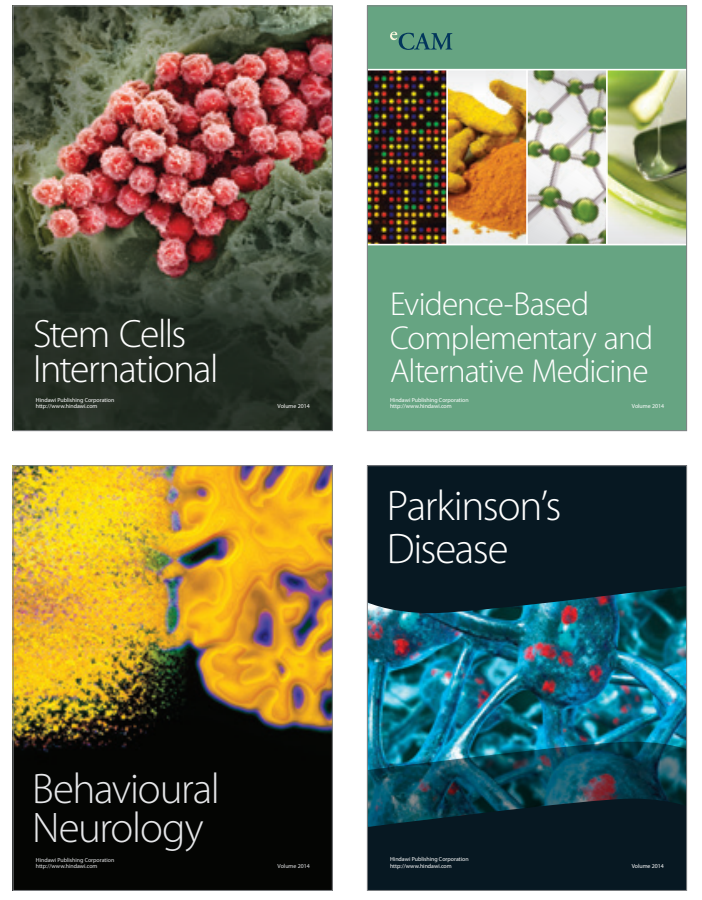
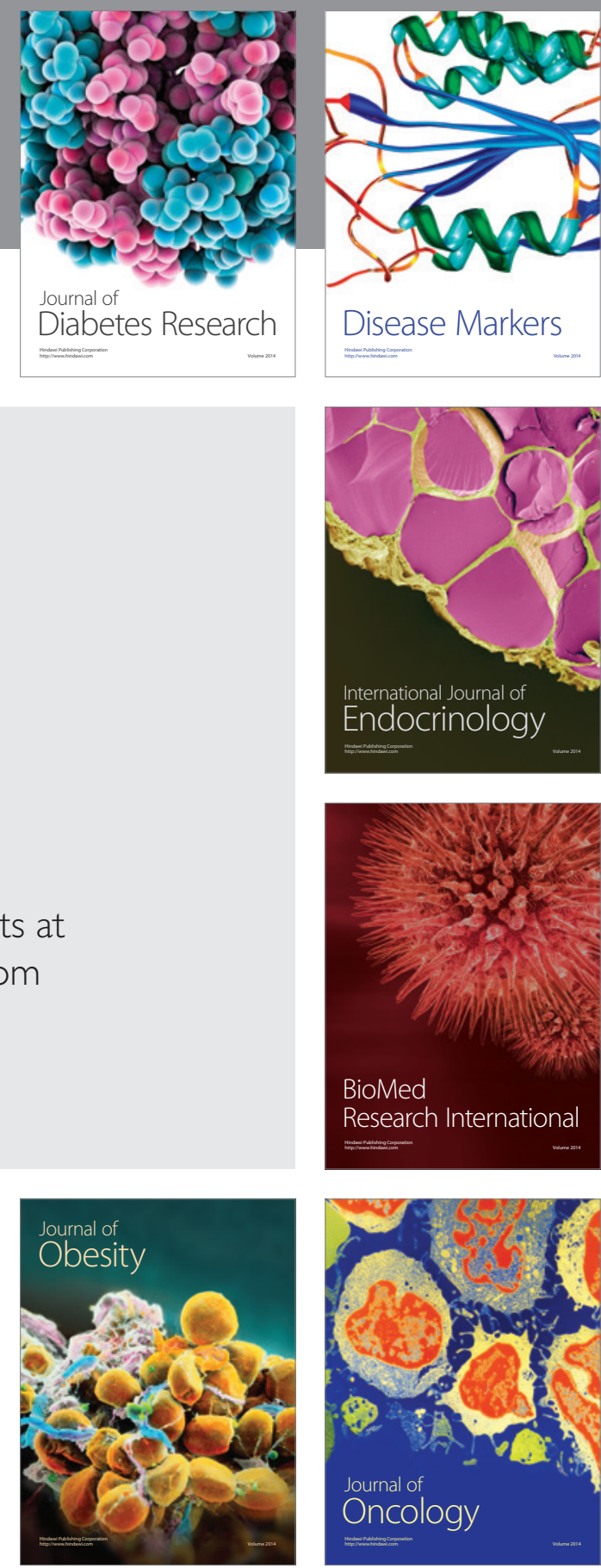

Disease Markers
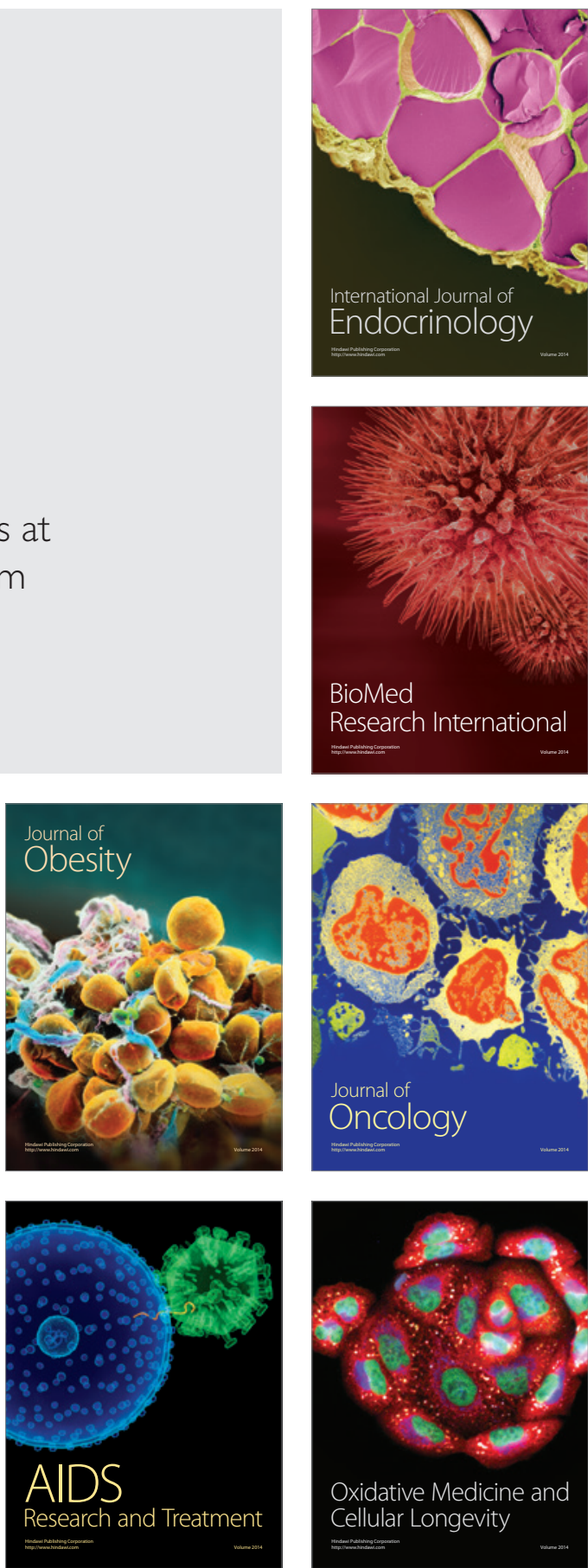